\title{
Social and economic influences on infant and child feeding practices in a Marshallese community
}

\author{
Britni L Ayers ${ }^{1}$, Marilou D Shreve ${ }^{2}$, Allison L Scott ${ }^{2}$, Victoria A Seaton ${ }^{3}$, Kelly V Johnson ${ }^{2}$, \\ Nicola L Hawley ${ }^{4}$, Brett Rowland', Ramey Moore ${ }^{5}$ and Pearl A McElfish ${ }^{1, *}$ \\ 'Office of Community Health and Research, University of Arkansas for Medical Sciences, Northwest Campus, 1125 \\ N. College Avenue, Fayetteville, AR 72701, USA: ${ }^{2}$ Eleanor Mann School of Nursing, University of Arkansas, \\ Fayetteville, AR, USA: ${ }^{3}$ Department of Pharmacy, University of Arkansas for Medical Science, Fayetteville, AR, USA: \\ ${ }^{4}$ Department of Chronic Disease Epidemiology, Yale School of Public Health, New Haven, CT, USA: ${ }^{5}$ University of \\ Arkansas, Fayetteville, AR, USA
}

Submitted 31 January 2018: Final revision received 24 July 2018: Accepted 10 December 2018: First published online 22 February 2019

\begin{abstract}
Objective: The present study aimed to examine the key influences on infant and child feeding practices among a Marshallese community at each social ecological level. It is the first study to examine the key influences on infant and child feeding practices with Marshallese immigrant women in the USA and helps fill a gap in the previous literature that has included other immigrant women.

Design: Community-based participatory research design with twenty-seven participants taking part in four qualitative focus groups.

Setting: The study took place within the Marshallese community in Arkansas, USA. Participants: Participants included Marshallese women with children aged 1-3 years and/or caregivers. Caregivers were defined as someone other than the parent who cares for children. Caregivers were often older women in the Marshallese community.

Results: There were five primary themes within multiple levels of the Social Ecological Model. At the intrapersonal level, mothers' and caregivers' autonomy emerged. At the interpersonal level, child-led and familial influences emerged. At the organizational level, health-care provider influences emerged; and at the policy level, the Special Supplemental Nutrition Program for Women, Infants, and Children emerged as the most salient influence.

Conclusions: Marshallese immigrant women's infant and child feeding practices are influenced at intrapersonal, interpersonal, organizational and policy levels. Understanding these multidimensional influences is necessary to inform the creation of culturally tailored interventions to reduce health disparities within the Marshallese community.
\end{abstract}

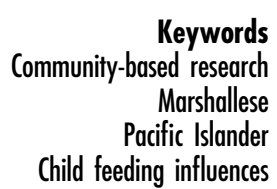

Obesity continues to rise in the USA despite decades of clinical approaches and community-based interventions. Current estimates indicate $39.8 \%$ of adults and $20.0 \%$ of children (aged 6-19 years) in the USA are obese (BMI $\geq$ $\left.30 \cdot 0 \mathrm{~kg} / \mathrm{m}^{2}\right)^{(1)}$. Arkansas has the fourth highest adult obesity prevalence in the nation, with adult obesity at $35.7 \%$ and childhood obesity (BMI $\geq 95$ th percentile) ranging from $14 \cdot 4 \%$ (age $1-4$ years) to $20 \cdot 0 \%$ (age $10-17$ years) ${ }^{(1)}$. US immigrants often face even higher prevalence of obesity $^{(2)}$. According to the 2003 New Immigrant Survey (NIS), more than $45 \%$ of new immigrants are either overweight or obese $^{(3)}$. Considering that immigrants comprise $12.5 \%$ of the total population and are increasing in population size, it is crucial to address immigrant obesity ${ }^{(3)}$.
Pacific Islanders face higher rates of obesity ${ }^{(4)}$ and obesity-related chronic conditions ${ }^{(5,6)}$. The Center for Disease Control and Prevention's National Health Interview Survey documented that $44 \%$ of Pacific Islanders surveyed in 2010 were obese, compared with $37 \%$ of African Americans and $32 \%$ of Hispanics/Latinos ${ }^{(4)}$. Northwest Arkansas is home to a large and growing Marshallese Pacific Islander community with an estimated 12000 Marshallese living in Arkansas compared with approximately 40000 residing in the $\mathrm{USA}^{(7,8)}$. The health disparities of Marshallese residing in north-west Arkansas are even more pronounced. A health screening study with Pacific Islanders in Arkansas revealed $90 \%$ of adult participants ( $n$ 401) were overweight or obese ${ }^{(9)}$. 
Research consistently demonstrates that exclusive breast-feeding decreases the risk of obesity and cardiometabolic disease for both mother and infant ${ }^{(10,11)}$ and may protect against obesity in children into adulthood ${ }^{(12)}$. Early introduction of formula or complementary foods and discontinuation of breast-feeding increase the risk of obesity and diabetes ${ }^{(13)}$. Further, as infants and children transition to the family diet, family diet preferences and practices exert an influence on children's preferences and consumption levels ${ }^{(14)}$. Available evidence shows that obesity risk among immigrant children is influenced by a number of personal and environmental factors including formula supplementation, early introduction of solid foods before 4 months of age, family's length of stay in the USA, changes in dietary and physical behaviours, and economic and social barriers ${ }^{(15-18)}$. However, there is little to no data on the key influences on infant and child feeding practices among Pacific Islander immigrants who have high obesity rates.

The Pacific Islander population in the USA is increasing, with significant and rapid growth in Southern states. Arkansas has seen a $252 \%$ growth in the Pacific Islander population between 2000 and 2010; the vast majority are from the Republic of the Marshall Islands ${ }^{(19)}$. The health disparities are embedded in a complex history between the USA and the Marshallese people. The US military conducted nuclear testing in the Marshall Islands between 1946 and 1958, detonating sixty-seven fission and thermonuclear devices equivalent to 7200 Hiroshima-sized bombs ${ }^{(20)}$. As a result, areas of the Marshall Islands were contaminated, disrupting their dominant food sources of fish and locally grown plants. The Marshallese lifestyle and diet shifted to a Western diet high in simple carbohydrates and fat and a more sedentary lifestyle after the era of nuclear testing. The majority of foods are now imported from outside the country $^{(6)}$. Rice is the primary staple food in addition to simple-carbohydrate foods such as ramen noodles (quick cooking noodles), doughnuts and pancakes. Consumption of fruits and vegetables is very low ${ }^{(21)}$. These same foods continue to be staples in their diet after migration to the USA due to their familiarity and low cost ${ }^{(22,23)}$. After the nuclear testing, the USA conducted research on exposed Marshallese to understand the effects of nuclear radiation on human subjects. The research was conducted without informed consent or information provided in the native language. As a result, the Marshallese community exhibits distrust of research due to this historical trauma ${ }^{(24,25)}$.

To overcome the challenges of this historical trauma, the University of Arkansas for Medical Science established a community-based participatory research (CBPR) partnership with the Marshallese community. CBPR is a research approach seeking to involve community partners in all aspects of the research process ${ }^{(26)}$. This type of research is uniquely suited for engaging indigenous and immigrant populations to overcome historical trauma ${ }^{(26,27)}$. The present study was developed based on a CBPR partnership focused on maternal and child health. The research was conducted through a collaboration of academic and Marshallese community researchers. The research question guiding the study was: What are the key influences on infant and child feeding practices within the Marshallese community? Understanding the influences will help inform interventions, practices and policies.

\section{Research design and methodology}

\section{Recruitment and sampling}

As part of the CBPR collaborative, the research team has spent the past five years meeting with the Marshallese community members to determine and prioritize the community's primary health concerns. The present study was developed after numerous group discussions which included academic and Marshallese community researchers. From these discussions, maternal and child health was determined a priority to addressing and preventing obesity and diabetes.

Participants were recruited through community organizations, specifically the Arkansas Coalition of Marshallese, pastors and community contacts developed through CBPR fieldwork. Specifically, participants were approached by bilingual community co-investigators. The inclusion criteria specified participants be Marshallese women with children aged 1-3 years and/or caregivers of those children. Caregivers were defined as someone other than the parent who cares for the child. Caregivers were often older women (called 'aunties') in the Marshallese community. Marshallese community co-investigators felt it was important to include caregivers because they have a significant influence over infant and child feeding practices within the Marshallese community.

\section{Theoretical framework}

The Social Ecological Model (SEM) considers the intrapersonal, interpersonal, organizational and policy influences on health and can be used to identify factors potentially amenable to intervention and modification ${ }^{(28)}$ (Fig. 1). The SEM was used as a guiding theoretical framework for the present study. The SEM acknowledges the interdependent, reciprocal and cumulative influence of factors at multiple levels including: (i) intrapersonal factors (individual autonomy); (ii) interpersonal factors (social networks); (iii) organizational factors (health-care services); (iv) and policy factors (policies affecting access to food) ${ }^{(28)}$. The SEM focuses on multiple pathways that may directly inform health promotion interventions and policies. 


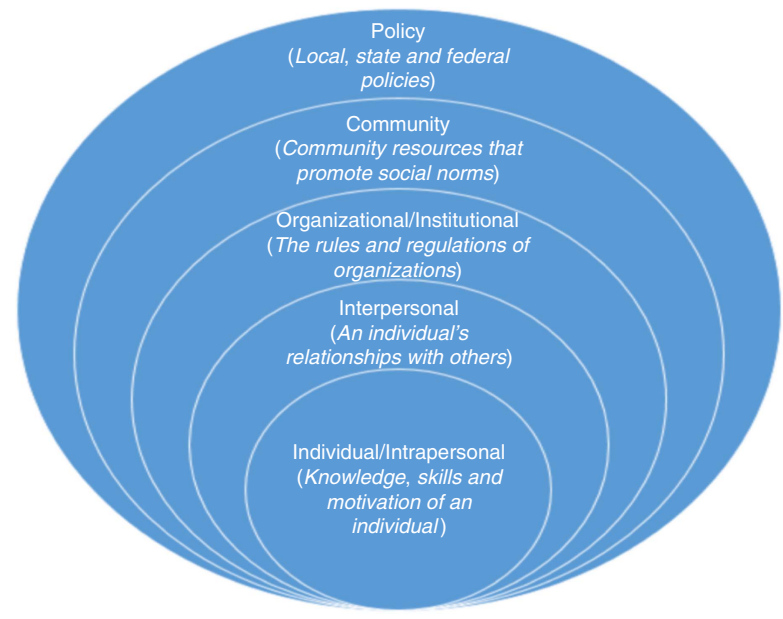

Fig. 1 (colour online) Social Ecological Model. Adapted from McLeroy et al. ${ }^{(56)}$

\section{Research design}

A qualitative descriptive design approach, using focus groups with Marshallese mothers and caregivers, was chosen to allow participants to use their own words to describe the key influences of their infant and child feeding practices. A qualitative descriptive design provides a summary of experiences and meanings participants ascribe to those experiences ${ }^{(29)}$. A semi-structured focus

\section{Table 1 Focus group discussion guide}

1. When, and if, you introduce solids to your baby, what made you decide it was time?

a. Who influenced you the most in this decision?

b. What did you give them first other than milk?

c. How did you decide what to give them first?

d. What are your thoughts about this?

2. When do you think is the best time to introduce solids?

a. Do members of your family agree with you on when to introduce solids to your child?

b. Are there certain foods that infants and small children should avoid?

3. What type of rice do you eat and how do you prepare it? a. How often do you eat rice? Your baby?

b. When did you or your caregiver first give rice to your children?

c. How do you prepare rice for your family?

d. Tell me more?

4. What fruits and vegetables do you eat? Your baby?

a. How often?

b. Who decides which fruits/vegetables to buy?

c. What prevents you and your family from eating more fruits/ vegetables?

5. What does your baby mainly drink?

a. Who decides what they drink?

b. Has WIC influenced this?

c. How much fruit juice does your baby drink?

6. Has WIC affected your feeding practices?

a. If so, how has this affected how you feed your baby?

b. Can you tell me more?

c. What are examples of changes you made after WIC?

d. What has WIC or your doctor told you about when and how to introduce solids to your baby?

7. Who selects and purchases the food that is eaten in your home? a. Who prepares the meals?

b. Who in your family eats first?

WIC, Special Supplemental Nutrition Program for Women, Infants, and Children. group guide with open-ended questions was used to encourage participants to speak candidly while maintaining consistent inquiries across the focus groups. The focus group discussion guide was developed in partnership with the CBPR team and through extensive fieldwork (Table 1). Broad questions were designed to encourage open dialogue. Probes were used to clarify nuances.

\section{Data collection}

From November 2016 to February 2017, a purposive sample of twenty-seven participants took part in four focus groups. Each focus group consisted of mothers and/ or caregivers. Based upon Marshallese community COinvestigators' input, the CBPR team included both mothers and/or caregivers within the focus groups. Marshallese community co-investigators stated that it was important to include caregivers because they have a significant influence over maternal care within the Marshallese community as the women collectively raise the children. Care was taken to ensure all participants' voices were heard. The group size ranged from five to ten with an average focus group size of seven participants. A female bilingual (Marshallese and English) community co-investigator, trained in research methods, facilitated each focus group. Focus groups took approximately $1 \mathrm{~h}$ and were conducted at the office of a community-based organizational partner. Participants were provided a meal and a \$US 25 gift card for their participation.

A summary of the project and consent form was reviewed by the participants; all materials were provided to the participants in Marshallese. After consent, participants completed a brief written survey that included questions on demographic characteristics (Tables 2 and 3).

\section{Data analysis}

Focus groups were audio-recorded and transcribed verbatim by a female bilingual community co-investigator.

Table 2 Participant demographics: mothers with children aged 1-3 years from the Marshallese community in Arkansas, USA, November 2016-February 2017

\begin{tabular}{lcc}
\hline & $n$ or mean & \% of sample* or SD \\
\hline Age (years) $\dagger$ & 31.0 & 7.97 \\
Received prenatal care (yes) & 13 & 100.0 \\
Length of time in USA (years) $\dagger$ & 9.29 & 6.74 \\
Currently enrolled in WIC (yes) & 8 & 61.5 \\
Breast-fed child (yes) & 13 & 100.0 \\
Supplemented with formula (yes) & 8 & 72.7 \\
Age at first solid food (months) $\dagger$ & 10.2 & 4.06 \\
First food introduced & & \\
$\quad$ Baby food & 5 & 45.5 \\
$\quad$ Banana & 3 & 27.3 \\
Other & 3 & 27.3 \\
\hline
\end{tabular}

WIC, Special Supplemental Nutrition Program for Women, Infants, and Children.

*Percentages are based on the number of valid responses for each item. Percentages may not total 100 due to rounding. †Mean and SD are reported for continuous variables. 
Table 3 Participant demographics: caregivers of children aged 1-3 years from the Marshallese community in Arkansas, USA, November 2016-February 2017

\begin{tabular}{lcc}
\hline & $n$ or mean & $\%$ of \\
\hline Age (years) $\dagger$ & $52 \cdot 8$ & 9.76 \\
Length of time in USA (years) $\dagger$ & 9.11 & 7.02 \\
Age at first solid food (months) $\dagger$ & 10.9 & 2.13 \\
First food introduced & & \\
Rice & 6 & 50.0 \\
Soup & 3 & 25.0 \\
Baby food & 2 & 16.7 \\
Other & 1 & 8.3 \\
\hline
\end{tabular}

*Percentages are based on the number of valid responses for each item. †Mean and SD are reported for continuous variables.

Transcripts were then translated from Marshallese to English and checked for accuracy by two female bilingual research staff. The CBPR team coded transcripts for emergent themes. All themes were collaboratively discussed to ensure scientific rigour, intercoder agreement and develop the most salient themes within the data. There were two primary coders and one confirmation coder. Codes were finalized and organized in a codebook. MAXQDA 12 software was used to manage the transcribed data $^{(30)}$. MAXQDA 12 was designed to facilitate and support qualitative, quantitative and mixed-methods data through a constant coding process. The qualitative results revealed four primary themes that emerged regarding the most key influences on infant and child feeding practices within the Marshallese community. Because the study uses the SEM as the theoretical framework, the emergent themes were organized into the SEM levels. Throughout the process, the Marshallese community co-investigators provided feedback to ensure that the nuanced meanings of participants' responses were captured.

\section{Results}

There were four focus groups conducted (denoted as FG1-FG4 below). After the first two focus groups, no new themes or sub-themes emerged suggesting saturation was reached. While the additional focus groups did not add new themes, they did continue to add richness to the data. Quotes from all focus groups are included. The mean age was 31.0 years for mothers and 52.8 years for caregivers. The average length of time living in the USA was just over 9 years for both mothers and caregivers. Every mother reported receiving prenatal care, and 61.5\% of participants were currently accessing the Special Supplemental Nutrition Program for Women, Infants, and Children (WIC).

All mothers reported breast-feeding their infants, and $80 \%$ reported using some type of milk supplementation. Mothers reported the mean age to introduce solids to be $10 \cdot 2$ months. Caregivers reported the appropriate age to introduce solids to infants was 8.18 months, but when asked what age the caregiver actually introduced solids the mean age was 10.9 months. Mothers reported that the most common first food introduced was baby food (45.5\%), whereas caregivers reported that the most common first food introduced was rice $(50 \cdot 0 \%)$.

\section{Influences on infant and child feeding practices}

Participants discussed the key influences on infant and child feeding practices within the Marshallese community. Themes emerged within multiple levels of the SEM. At the intrapersonal level, mothers' and caregivers' autonomy emerged. At the interpersonal level, child-led and familial influences emerged. At the organizational level, healthcare provider influences emerged, and at the policy level, WIC influences emerged.

\section{Intrapersonal influences}

\section{Mothers' and caregivers' autonomy}

Mothers and caregivers discussed how they perceived themselves as having a significant degree of autonomy in decisions related to infant and child feeding practices. One participant said:

'As for me, I am the one that chooses when my child is ready to eat regular food. If the time comes for them to start eating regular food then I will feed them. My family members have no say in when I choose to feed my children regular food.' (FG3)

Another participant said:

'No one did bother with what I have to do with my children.' (FG4)

Participants agreed and said that:

'No one minds what I do to my kids, it is just me.' (FG4)

When we specifically asked about who or what influenced their infant and child feeding practices, one participant said:

'We already know [what] we are required to do. Like breast-feeding, they show us what to do, but I was already doing them.' (FG3)

Participants agreed that it was the role of the female of the house to determine these practices. When asked about the role of fathers in this process, one participant stated:

'His job is to give me money so I can go purchase foods.' (FG4)

Overall, participants stated:

'It is usually us mothers who decide.' (FG3) 


\section{Interpersonal influences}

While mothers discussed being the primary decision maker in infant feeding, participants also discussed interpersonal relationships as being highly influential in their infant and child feeding practices. Within the interpersonal influences theme two sub-themes emerged: (i) child-led influence and (ii) familial influence.

\section{Child-led influence}

Participants described the perceived desires and needs of their infants and children as having an especially strong influence on infant and child feeding practices. Much of the discussion surrounding breast-feeding supplementation was predicated on child-led feeding. One caregiver participant described how her daughter (the infant's mother) decided when to introduce solid foods, saying:

'She started giving her child solid food when the child was 3 months old. She did so to make the child's tummy fully satisfied which prevents the child from being fussy, and then child would be full for a while.' (FG1)

Child-led feeding practices not only determined when solid food was introduced, but also what foods were introduced. One participant said:

'Usually, some of our children while growing up, they would usually avoid vegetables.' (FG1)

Participants described children as being very influential in what food choices were made for their families and exerting powerful influence over the food choices. As one participant said:

'If we do not get what they want they will scream their heads off inside the store stating that the WIC or SNAP [Supplemental Nutrition Assistance Program] benefit is theirs.' (FG1)

Participants described that, overall,

'The children buys [chooses] the foods and the parents prepare them.' (FG1)

\section{Familial influence}

Participants discussed the pervasiveness of familial influence on their infant and child feeding practices. The majority of participants, both mothers and caregivers, described turning to elders and family when deciding when and what foods to introduce to their infants. One mother said:

'My mom instructed me to start feeding my child.' (FG2)

Another mother described relying on both parents for infant feeding advice when she stated:
'Since it was our first child, and we have no clue as to what to do. So they [parents] were the one that direct us.' (FG2)

The discourse on familial influence went beyond instructions from the mothers' parents. One participant described getting information

'From our mom, dad, grandma, and the older ladies; they would stick by our side and assist.' (FG1)

Another participant stated:

'When someone is a single mom and needs help our family would help us out with what we do.' (FG1)

Participants described a communal effort to not only help mothers know what to feed, but also would supply food if needed. As one participant described:

'As we are all aware that us Marshallese have good hearts and will not watch one suffer. They would help us out.' (FG1)

The overall sentiment from the mothers and caregivers was:

'You know how Marshallese are, we ask each other. Well of course, that is part of our culture, when we do not have enough, we do what we usually do.' (FG4)

\section{Organizational influences}

Health-care providers' influence

Mothers and caregivers differed on their views of healthcare providers' influence on infant and child feeding practices. Majority of the caregivers described health-care providers as influential on when and what food to introduce, both in the Marshall Islands and in the USA. For example, one caregiver said:

'I have a child that lives in the Marshall Islands, when I used to go to my check-up appointments, the doctors usually says, "When the child turns 6 months old, you can start feeding her solid foods but use the blender to soften up the foods, make sure to cook them so they are soft; for instance, if it is a rice make sure to make it soft to a point where it is like a soup so the child has no problem eating it".' (FG4)

Caregivers discussed health-care providers as being helpful despite their economic means, and caregivers discussed health-care providers with high regard, respect and deference. For example, one caregiver stated:

'They [health-care providers] are the ones that educate and encourage us. We have to give them praises for even though they did not have enough resources back then they were doing all their best with us. For those of us that lived in those times, 
financial assistance was scarce to our children. And we ought to give them praises for all they have done. They would advise us to feed our children this and that.' (FG1)

The mothers' discourse varied on whether they thought health-care professionals were influential. Some mothers described the experience as encouraging. For example, one mother described:

'Here in the States, the doctors are the ones that encourage us to start feeding our children.' (FG2)

Meanwhile other mothers described health-care providers as not being influential on how to feed their infants and said:

'The doctor place, they do not ask you how the child is fed? They do not tell you how to feed them.' (FG3)

Another mother described fear in following her healthcare provider's suggestions on when to introduce solids when she said:

'There are times when the doctors tell us to start the feeding process, but we are afraid they might choke.' (FG2)

Lastly, one mother described relying on previous observations rather than health-care provider's advice when she said:

'So we do not have to follow what others do since we saw what our parents did while growing up, and we learned by observing. So for us, I do not believe we need to go to a doctor and that doctor will say what we need to do.' (FG4)

\section{Policy influences}

At the policy level, the influence of WIC on Marshallese participants' infant and child feeding practices was discussed and WIC was the most pervasive theme within the interviews. There were three sub-themes that emerged regarding WIC including: (i) WIC's influence on breastfeeding and/or formula-feeding; (ii) WIC's influence on introduction of complementary foods; and (iii) limitations of WIC to meet infant and child feeding needs.

WIC's influence on breast-feeding and/or formula-feeding WIC was described as influential on participants' infant feeding practices regarding both breast-feeding and formula-feeding. WIC was discussed most in the context of working outside the home and WIC's support for pumping breast milk and providing free formula. For example, one participant said:

'When I went to WIC ... They told me to pump at times that I am at work so I can store the milk.' (FG3)

Another participant stated:
'The good thing about it [WIC] is that it helps us. When we go to work, child can have formulas but when we are at home we breast-feed them.' (FG1)

Participants discussed using formula because

'You get free milk [formula] from the WIC programme.' (FG4)

Although the participants described utilizing free formula as a positive benefit, there was negative discourse about the effect this had on breast-feeding. As one participant said:

'The] reason why it is bad is because the new mothers, the young mothers, depend a lot on the milk [formula] rather than breast-feed their children.' (FG1)

WIC's influence on introduction of complementary foods WIC was described as influential on both when and what complementary foods were introduced via nutrition classes, food vouchers and the food being provided. As one participant stated:

'There was one point that I attended a nutrition class, and I was told that once my child reach 6 months she can start taking food. When my child turns 6 [months], I will begin to receive WIC voucher which I can use to purchase my baby foods with. And I was also told that I can start feeding my child solid foods. And I got this information out of the WIC programme. But I have not got any information from his doctor since he is not due back yet. This was the information I received from the WIC programme on when to introduce solid food to my child.' (FG1)

Participants discussed the introduction of complementary food education they received and the effect this had on detecting food allergies, with one stating:

'I watched a video at the WIC place, just like how she said, to introduce one food at a time, you cannot mix the food with another, so that you can know what your child is allergic to. And it also showed ways on how to feed them, how much you should give the baby, and this movie explained it.' (FG3)

Limitations of WIC to meet infant and child feeding needs The overall discourse about WIC was that it was influential and helpful in providing food vouchers for healthy food. However, the participants discussed that WIC's provisions were not enough for their families' needs, and they were still challenged to get enough formula and healthy food. For example, one participant said:

'It could only last a day. It could be just one day, and everything is gone.' (FG4) 
Another participant continued:

'Yes, for my grandchildren, when they drink the WIC milk [formula], it only last two weeks, they do not last a month, it is not enough for a month, sometimes I have to go around and see where I can get some more.' (FG4)

Participants discussed the provisions of WIC running out and limiting their consumption of fresh fruits and vegetables. One participant said:

'The thing that prevent some families from eating fruits and greens is because there is no money, and it is not time for WIC yet.' (FG3)

Another participant agreed and stated:

'When there are fruits, they eat them ... and when there is no more, that is when they stop, until the next WIC is, we go and get some more. Or we wait until we have enough money to go and buy.' (FG3)

Participants not only described relying on WIC for healthy food options but also discussed the economic inability to provide fresh fruits and vegetables for their families beyond WIC vouchers. As one participant said:

'They only eat when we have WIC for them and when it is out, we wait until there is money to buy.' (FG3)

Another participant said:

'The moment you ran out of fruits and vegetables that is when you stop eating it.' (FG2)

Participants stated that:

'There is no money to purchase more so we cannot eat more.' (FG2)

Overall, participants described the inadequate WIC supply for the clan-like family as highly influential in children's limited consumption of healthy food, and most specifically fruits and vegetables. One participant said:

'The child, they stop because the fridge is empty but if there are some [fruits and vegetables], they will keep eating and eating.' (FG4)

\section{Discussion}

The purpose of the present study was to examine the key influences on infant and child feeding practices among a Marshallese community at each social ecological level. Although there have been previous studies with immigrant women to examine influences on infant and child feeding, the present study is the first to examine these influences with Marshallese immigrant women in the USA ${ }^{(31-33)}$. The SEM provided a valuable framework for examining the effects of the interpersonal, intrapersonal, organizational and policy levels that influence infant and child feeding practices among Marshallese immigrant women.

A novel finding of the study was the mothers' and caregivers' perception of autonomy in making decisions regarding infant and child feeding practices despite the collectivist culture of the Marshallese community. Participants were quite confident in their individual decision regarding child feeding practices, and sometimes described being the sole, autonomous influence on what and when their infant ate. Although individual influence was discussed, the discourse was collectivist in nature with phrases such as 'us mothers decide'. Much of the discussion differentiated their decision from outside influence from health-care providers. This finding is not uncommon among other immigrant women wherein mothers view themselves as being solely responsible for children's eating and feeding behaviours ${ }^{(34)}$. In addition, participants described child-led influence on feeding practices wherein children determined when and what foods to eat, even if these were unhealthy choices. This finding aligns with previous studies with immigrant families wherein mothers reported being highly responsive to children's requests for unhealthy food $^{(35,36)}$. Research among Brazilian, Haitian and Latina women demonstrates that low-income immigrant mothers consistently have a high responsive feeding style to their child's needs ${ }^{(37,38)}$. The causes of this high responsive feeding style to immigrant children are not well understood and merit further research.

An anticipated finding was the confirmation of familial influence on infant and child feeding practices. Prior literature has documented that the Marshallese community is very family-oriented ${ }^{(39,40)}$. However, familial influences extended well beyond what complementary foods to introduce and when. Participants described relying on extended family and community members for additional provisions when food, money and/or WIC rations were low. This extension of familial influence was described by participants as 'part of our culture'. These findings are similar to previous literature that identified $25 \%$ of Marshallese living on the Marshall Islands share their US Department of Agriculture commodity foods with extended family and community members ${ }^{(21)}$. These cultural practices are indicative of collectivist food sharing identified in other literature on Pacific Islander communities ${ }^{(22,41-43)}$. Evidence of multigenerational female influence on breast-feeding and child feeding behaviour is documented among other immigrant communities globally, suggesting that interventions to target childhood obesity need to consider the role and influence of the extended family ${ }^{(44,45)}$, especially among Pacific Islander communities $^{(46,47)}$.

At the organizational level, caregivers described healthcare providers as more influential in providing information on infant feeding, and when and what complementary foods to introduce to infants, compared with the mothers. 
However, caregivers seem to be referring to health-care providers within the Marshall Islands and it is unclear if this influence is present in the USA. Research demonstrates that racial/ethnic minority and/or immigrant women experience fear, perceptions of mistreatment and have an overall lack of trust of health-care providers ${ }^{(48-50)}$. Further, this generational discord may be indicative of the linguistic and/or cultural barriers that health-care providers may exhibit within the USA for Marshallese mothers ${ }^{(51)}$. Previous research with Latina immigrant mothers has described participants' desire to have educational information from health-care providers but in their native language ${ }^{(34,52,53)}$. Despite this disharmony, older women are held in high esteem within the Marshallese culture and, therefore, caregivers may still provide a strong influence in viewing health-care providers as an influential resource on infant and child feeding information.

There was significant discussion regarding WIC as a policy-level influence. Participants viewed the nutrition education, food vouchers, breast pumps, formula and advice for infant and child feeding from WIC as positive. The breast pumps and formula were described as particularly important for mothers who worked outside the home. Prior literature also shows immigrant mothers working outside the home more after moving to the USA, which constrains their ability to breast-feed ${ }^{(47)}$. This is also consistent with previous research that identified the influential role of WIC in providing parental education and healthy food options to low-income Latina immigrant women $^{(34,52,53)}$. Although participants in the present study described WIC's free formula as helpful economically, they also expressed concern for how this deterred breastfeeding for new mothers. Previous studies with immigrant women, including Marshallese, have identified successive generations are utilizing formula rather than breast-feeding once they come to the USA, and the literature attributes this decline in breast-feeding as due in part to the free formula from WIC $^{(47,54)}$. WIC does provide an increased voucher for food if participants exclusively breast-feed their infants, but the present study participants did not mention this option and may be unaware of this opportunity. This dichotomy merits further investigation. It is important to understand how influential WIC is in deterring breast-feeding in immigrant families. WIC education and food vouchers did appear to have a positive influence on when and what foods were offered to children. Particularly the mothers discussed following the guidelines from WIC on which foods to introduce and when to introduce those foods. Interestingly, both mothers and caregivers described a late onset of the introduction of complementary foods compared with other immigrant families ${ }^{(15,17,18)}$.

Participants described WIC's provisions as not being substantial enough for their clan-like families. The inability of WIC's provisions to support Marshallese families may be indicative of the cultural difference between Western individualistic and Marshallese collectivist definitions of family, and therefore family size. For example, the definition of family in the Marshallese community typically includes multiple extended biological and nonbiologically affiliated community members who may live in a household ${ }^{(55)}$. This variance in the definition of family may not be understood in WIC's policies for allocation of provisions based on family size. Further, there may be a misunderstanding in viewing WIC's provisions as fully sufficient for feeding their family rather than supplemental. However, similar to Marshallese families in the Marshall Islands, there was discussion of sharing WIC provisions within the Marshallese community when food supplies were low, potentially demonstrating collectivist food sharing ${ }^{(21)}$.

\section{Study strengths and limitations}

The limitations of the present study included a non-random, small sample size from a single geographic area. While the sample size allowed us to reach saturation and was appropriate for the exploratory study, the findings may not be generalizable to other Pacific Islanders outside Arkansas. Despite the limitations, the study contributes to the gap in the literature on the key influences on infant and child feeding practices within a Marshallese community in the USA. The inclusion of caregivers and mothers is demonstrated as a strength to the study and provided insight into the dietary acculturation and generational variances on the key influencers of infant and child feeding practices among the participants.

\section{Practice and policy implications}

Understanding the key influences on infant and child feeding practices informs interventions, practices and policies. Participants described the strong influence of family in their feeding practices, whether this was determining when and what complementary foods to introduce, or for additional food provisions. Educational interventions should focus on family and caregivers to have the greatest effect. Caregivers viewed health-care providers as a positive influence on infant and child feeding practices. Given the value placed on older women's opinions, health-care providers need to be encouraged to talk about infant and child feeding practices more with their Marshallese patients. At the policy level, it appears that although WIC provided support via nutrition classes, breast pumps, formula, food vouchers and the food being provided, this was not perceived as adequate for Marshallese families. Further, the lack of discussion about breast-feeding incentives that WIC provides suggests the need to inform the community of these opportunities. Additionally, incorporating more information on community resources such as local food pantries and community gardens during the distribution of WIC could be beneficial, because it appeared that fruits and vegetables are highly desired within the Marshallese 
community but participants reported not being able to purchase them.

\section{Conclusion}

The present study is the first to explore the key influencers of infant and child feeding practices among Marshallese immigrant women in the USA. It is an important addition to the gap in the literature as it provides insight into a culture with high rates of obesity and cardiometabolic health disparities. The study results indicated that multiple intrapersonal-, interpersonal-, organizational- and policylevel factors influence Marshallese immigrant women's infant and child feeding practices. Understanding these multidimensional influences is necessary to inform and create culturally tailored interventions to reduce health disparities within the Marshallese community.

\section{Acknowledgements}

Financial support: The CBPR partnership support was provided from the University of Arkansas for Medical Sciences Translational Research Institute (grant number UL1TR000039), which was funded through the National Institutes of Health's National Center for Research Resources and National Center for Advancing Translational Sciences. Grant funding (\$US 1200) was received from Sigma Theta Tau Pi Theta Chapter (grant number 0402-82275-21-000). In addition, the CERT programme was funded by the Patient Centered Outcomes Research Institute (PCORI) (grant number \#1513-UAMS). Research reported in this publication was also partially supported by the National Institute of General Medical Sciences of the National Institutes of Health (award number P20GM109096). The content is solely the responsibility of the authors and does not necessarily represent the official views of the National Institutes of Health. Neither the University of Arkansas for Medical Sciences Translational Research Institute, which was funded through the National Institutes of Health's National Center for Research Resources and National Center for Advancing Translational Sciences, nor the Sigma Theta Tau Pi Theta Chapter, nor the National Institute of General Medical Sciences of the National Institute of Health had a role in the design, analysis or writing of this article. Conflict of interest: None. Authorship: K.V.J., A.L.S., M.D.S. and P.A.M. formulated the research questions and design of the study. K.V.J., A.L.S., M.D.S., V.A.S. and B.L.A. implemented the study. Data were analysed by M.D.S., V.A.S., B.R. and B.L.A. B.L.A. and P.A.M. wrote the manuscript. R.M., K.V.J., A.L.S., M.D.S., V.A.S. and N.L.H. edited the manuscript. Ethics of human subject participation: This study was conducted according to the guidelines laid down in the Declaration of Helsinki and all procedures involving human subjects were approved by the University of Arkansas' Institutional Review Board (\#16-07-010). Informed consent was obtained from all subjects and formally recorded.

\section{References}

1. Hales C, Carroll M, Fryar CD et al. (2015) Prevalence of obesity among adults and youth: United States, 2015-2016. Key findings. Data from the National Health and Nutrition Examination Survey. https://www.cdc.gov/nchs/data/ databriefs/db288.pdf (accessed January 2019).

2. Choi JY (2012) Prevalence of overweight and obesity among US immigrants: results of the 2003 New Immigrant Survey. J Immigr Minor Health 14, 1112-1118.

3. Jasso G, Massey D, Rosenzweig M et al. (2006) The New Immigrant Survey 2003 Round 1 (NIS-2003-1) Public Release. Date March 2006. Funded by NIH HD33843, NSF, USCIS, ASPE \& Pew. http://nis.princeton.edu (accessed December 2018).

4. Schiller J, Lucas J, Ward B et al. (2012) Summary health statistics for US adults: National Health Interview Survey, 2010. Vital Health Stat 10 issue 252, 1-207.

5. Okihiro M \& Harrigan R (2005) An overview of obesity and diabetes in the diverse populations of the Pacific. Ethn Dis 15, S5-71-S5-80.

6. Hawley N \& McGarvey S (2015) Obesity and diabetes in Pacific Islanders: the current burden and the need for urgent action. Curr Diab Rep 15, 29.

7. Arkansas Department of Education Data Center (2016) Springdale School District Enrollment by Race, 2016-2017. Little Rock, AR: Arkansas Department of Education.

8. McElfish P (2013) Interview with Carmen Chong-Gum. Springdale, AR: Arkansas Marshallese Consulate.

9. McElfish P, Rowland B, Long C et al. (2017) Diabetes and hypertension prevalence in Marshallese adults: results from faith-based health screening events. J Racial Ethn Health Disparities 4, 1042-1050.

10. Schwarz EB, Ray RM, Stuebe AM et al. (2009) Duration of lactation and risk factors for maternal cardiovascular disease. Obstet Gynecol 113, 974-982.

11. Ram KT, Bobby P, Hailpern SM et al. (2008) Duration of lactation is associated with lower prevalence of the metabolic syndrome in midlife - SWAN, the study of women's health across the nation. Am J Obstet Gynecol 198, 268. e1-268.e6.

12. Arenz S, Rückerl R, Koletzko B et al. (2004) Breast-feeding and childhood obesity - a systematic review. Int J Obes Relat Metab Disord 28, 1247-1256.

13. Centers for Disease Control and Prevention, Department of Health and Human Services (2017) Breastfeeding Rates: National Immunization Survey (NIS). http://www.cdc.gov/ breastfeeding/data/nis_data/index.htm (accessed January 2019).

14. Thompson AL (2013) Intergenerational impact of maternal obesity and postnatal feeding practices on pediatric obesity. Nutr Rev 71, Suppl. 1, S55-S61.

15. Kaiser LL, Aguilera AL, Horowitz M et al. (2015) Correlates of food patterns in young Latino children at high risk of obesity. Public Health Nutr 18, 3042-3050.

16. Roshania R, Narayan KM \& Oza-Frank R (2008) Age at arrival and risk of obesity among US immigrants. Obesity (Silver Spring) 16, 2669-2675.

17. Wiley JF, Cloutier MM, Wakefield DB et al. (2014) Acculturation determines BMI percentile and noncore food intake in Hispanic children. J Nutr 144, 305-310.

18. Huh SY, Rifas-Shiman SL, Taveras EM et al. (2011) Timing of solid food introduction and risk of obesity in preschoolaged children. Pediatrics 127, e544-e551. 
19. Hixson L, Hepler B \& Kim M (2012) The Native Hawaiian and Other Pacific Islander Population 2010. Washington, DC: US Census Bureau.

20. Barker H (2012) Bravo for the Marshallese: Regaining Control in a Post-Nuclear, Post-Colonial World. Belmont, CA: Cengage Learning.

21. Gittelsohn J, Haberle H, Vastine A et al. (2003) Macro- and microlevel processes affect food choice and nutritional status in the Republic of the Marshall Islands. J Nutr $\mathbf{1 3 3}$, issue $1,310 \mathrm{~S}-313 \mathrm{~S}$.

22. Hallgren E, McElfish P \& Rubon-Chutaro J (2015) Barriers and opportunities: a community-based participatory research study of health beliefs related to diabetes in a US Marshallese community. Diabetes Educ 41, 86-94.

23. McElfish P, Hallgren E, Henry L et al. (2016) Health beliefs of Marshallese regarding type 2 diabetes. Am J Health Behav 40, 248-257.

24. Minkler M (2004) Ethical challenges for the 'outside' researcher in community-based participatory research. Health Educ Behav 31, 684-697.

25. Wallerstein N (1999) Power between evaluator and community: research relationships within New Mexico's healthier communities. Soc Sci Med 49, 39-53.

26. Vaughn LM, Jacquez F, Lindquist-Grantz R et al. (2017) Immigrants as research partners: a review of immigrants in community-based participatory research (CBPR). J Immigr Minor Health 19, 1457-1468.

27. Townsend C, Dillard A, Hosoda K et al. (2015) Communitybased participatory research integrates behavioral and biological research to achieve health equity for Native Hawaiians. Int J Environ Res Public Health 13, 4.

28. Gregson J, Foerster S, Orr R et al. (2001) Systems, environmental, and policy changes: using the social-ecological model as a framework for evaluating nutrition education and social marketing programs with low-income audiences. J Nutr Educ 33, Suppl. 1, S4-S15.

29. Neergaard MA, Olesen F, Andersen RS et al. (2009) Qualitative description - the poor cousin of health research? BMC Med Res Methodol 9, 52.

30. VERBI GmbH (1989-2015) MAXQDA, Software for Qualitative Data Analysis. Berlin: VERBI GmbH.

31. Ayala GX, Baquero B \& Klinger S (2008) A systematic review of the relationship between acculturation and diet among Latinos in the United States: implications for future research. J Am Diet Assoc 108, 1330-1344.

32. Birch LL \& Davison KK (2001) Family environmental factors influencing the developing behavioral controls of food intake and childhood overweight. Pediatr Clin North Am 48, 893-907.

33. Kumanyika SK (2008) Environmental influences on childhood obesity: ethnic and cultural influences in context. Physiol Behav 94, 61-70.

34. Lindsay AC, Machado MT, Sussner KM et al. (2008) Infantfeeding practices and beliefs about complementary feeding among low-income Brazilian mothers: a qualitative study. Food Nutr Bull 29, 15-24.

35. Evans A, Seth JG, Smith S, Harris KK et al. (2011) Parental feeding practices and concerns related to child underweight, picky eating, and using food to calm differ according to ethnicity/race, acculturation, and income. Matern Child Health J 15, 899-909.

36. Hennessy E, Hughes SO, Goldberg JP et al. (2012) Permissive parental feeding behavior is associated with an increase in intake of low-nutrient-dense foods among American children living in rural communities. J Acad Nutr Diet 112, 142-148.

37. Tovar A, Hennessy E, Pirie A, et al. (2012) Feeding styles and child weight status among recent immigrant motherchild dyads. Int J Behav Nutr Phys Act 9, 62.
38. Tovar A, Hennessy E, Must A et al. (2013) Feeding styles and evening family meals among recent immigrants. Int $J$ Behav Nutr Phys Act 10, 84.

39. McElfish P, Bridges M, Hudson J et al. (2015) Family model of diabetes education with a Pacific Islander community. Diabetes Educ 41, 706-715.

40. Yeary KHK, Long CR, Bursac Z et al. (2017) Design of a randomized, controlled, comparative-effectiveness trial testing a Family Model of Diabetes Self-Management Education (DSME) vs. standard DSME for Marshallese in the United States. Contemp Clin Trials Commun 6, 97-104.

41. Novotny R, Vijayadeva V, Grove J et al. (2012) Dietary intake among Native Hawaiian, Filipino, and White children and caregivers in Hawai'i. Hawaii J Med Public Health $\mathbf{7 1}$, 353-358.

42. Aitaoto N, Campo S, Snetselaar LG et al. (2015) Formative research to inform nutrition interventions in Chuuk and the US Pacific. J Acad Nutr Diet 115, 947-953.

43. Aitaoto NT, Braun KL, Ichiho HM et al. (2005) Diabetes today in the Pacific: reports from the field. Pac Health Dialog 12, 124-131.

44. Aubel J, Touré I \& Diagne M (2004) Senegalese grandmothers promote improved maternal and child nutrition practices: the guardians of tradition are not averse to change. Soc Sci Med 59, 945-959.

45. Aubel J (2012) The role and influence of grandmothers on child nutrition: culturally designated advisors and caregivers. Matern Child Nutr 8, 19-35.

46. Tapera R, Harwood M \& Anderson A (2017) A qualitative Kaupapa Māori approach to understanding infant and young child feeding practices of Māori and Pacific grandparents in Auckland, New Zealand. Public Health Nutr 20, 1090-1098.

47. Scott A, Shreve M, Ayers B et al. (2016) Breast-feeding perceptions, beliefs and experiences of Marshallese migrants: an exploratory study. Public Health Nutr 19, 3007-3016.

48. Wheatley RR, Kelley MA, Peacock N et al. (2008) Women's narratives on quality in prenatal care: a multicultural perspective. Qual Health Res 18, 1586-1598.

49. Attanasio L \& Kozhimannil KB (2015) Patient-reported communication quality and perceived discrimination in maternity care. Med Care 53, 863-871.

50. Sword W, Heaman MI, Brooks S et al. (2012) Women's and care providers' perspectives of quality prenatal care: a qualitative descriptive study. BMC Pregnancy Childbirth 12, 29.

51. Ayers BL, Hawley NL, Purvis RS et al. (2018) Providers' perspectives of barriers experienced in maternal health care among Marshallese women. Women Birth 31, e294-e301.

52. Sherry B, McDivitt J, Birch LL et al. (2004) Attitudes, practices, and concerns about child feeding and child weight status among socioeconomically diverse white, Hispanic, and African-American mothers. J Am Diet Assoc 104, 215-221.

53. Crawford PB, Gosliner W, Anderson C et al. (2004) Counseling Latina mothers of preschool children about weight issues: suggestions for a new framework. J Am Diet Assoc 104, 387-394.

54. Kimbro RT, Lynch SM \& McLanahan S (2008) The influence of acculturation on breastfeeding initiation and duration for Mexican-Americans. Popul Res Policy Rev 27, 183-199.

55. Berman E (2014) Holding on: adoption, kinship tensions, and pregnancy in the Marshall Islands. Am Anthropol 116, 578-590.

56. McLeroy KR, Bibeau D, Steckler A et al. (1988) An ecological perspective on health promotion programs. Health Educ Q 15, 351-377. 